\title{
Design of Hybrid Energy Management System for Wireless Sensor Networks in Remote Areas
}

\author{
Dr. Subarna Shakya \\ Professor, Department of Electronics and Computer Engineering, \\ Central Campus, Institute of Engineering, Pulchowk, \\ Tribhuvan University, \\ Pulchowk, Lalitpur Nepal. \\ Email: drss@ioe.edu.np.
}

\begin{abstract}
Energy is an essential parameter for any work to be done. An electric energy is a primary requirement for any wireless transmission system. Transmitting the information from a source to destination requires a continuous and strengthen wireless network connection to avoid interruption from electromagnetic disturbance. Powering such wireless networks can be done by giving direct current through a battery or from direct supply. The challenge comes when the wireless sensor network is fixed in a remote place or designed as a moving system for sensing analysis. Battery sourcing power system will be a better choice for such movable wireless system. The paper proposes a hybrid energy management system for wireless sensor networks (WSNs) connected in remote areas and it analyses the performance of the energy management system in terms of amount of energy can be generated for a battery source.
\end{abstract}

\section{Introduction}

WSNs are widely used in many applications like monitoring the environment changes, weather variations, animal tracking systems, agriculture farming system, and natural resource quality checking system and so on. By providing an independent power source to the WSNs, it can be placed anywhere in the world for collecting data about the fixed system. Battery replacement technology [1] is a simple technology used for identifying the status of battery power backup remotely from the destination point where the WSNs matters are received. With help of the received information about the battery status a maintenance team or person can visit the place to replace the battery source for continuous work. To carryout continuous operation of the remotely placed network an additional battery source will be provided. But the real challenge in the WSN is faster drain of battery source due to continuous mode of operation. A very good WSN system must work continuously without any delay, and to be independent in collecting data.

The necessity for designing a hybrid energy management system is to make the WSNs to operate without any disturbance due to lack of energy supply to the sensor nodes. There are several independent 
Journal of Electrical Engineering and Automation (EEA) (2020)

Vol.02/ No. 01

Pages: $13-24$

https://www.irojournals.com/iroeea

DOI: https://doi.org/10.36548/jeea.2020.1.002

energy harvesting systems are there which are suitable for only certain applications. The proposed hybrid energy management system is designed to satisfy all range of WSN applications. The figure 1 indicates the present available sources of energy materials to harvest energy for WSNs in a cost effective and maintenance free manner.

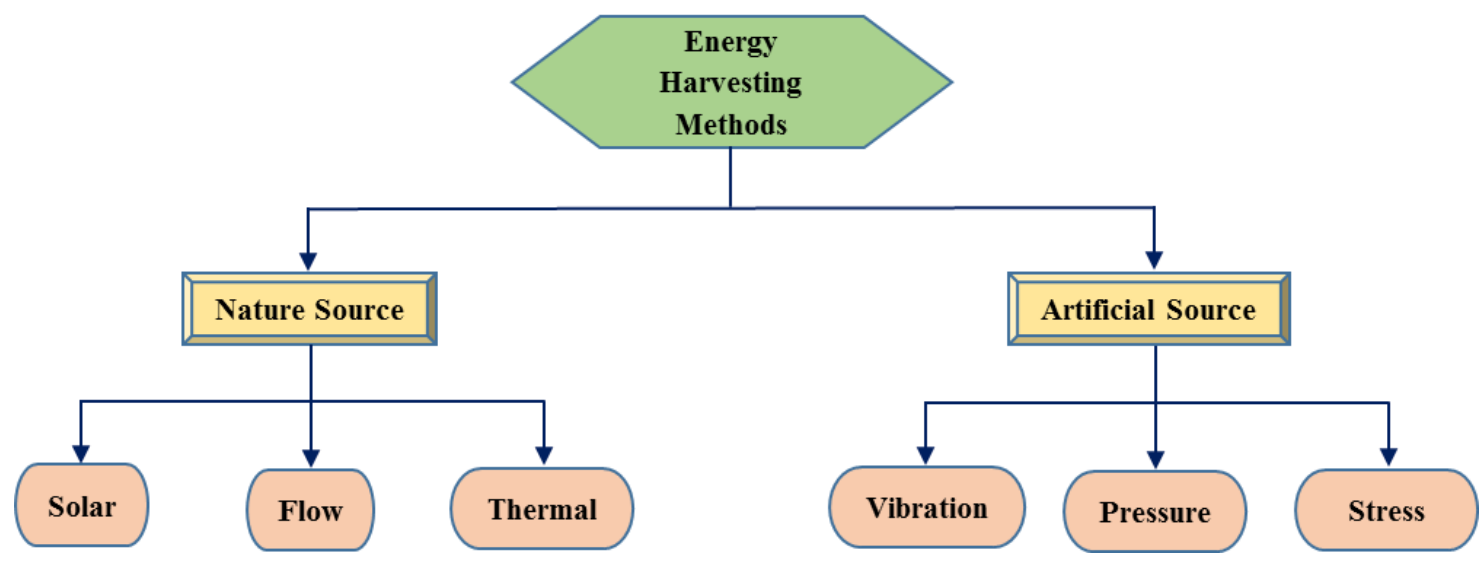

Figure 1. Energy harvesting methods for WSNs

The flow kind of natural energy consists of hydroelectric power generation and wind electric power generation. Energy can be harvested by any of these methods for WSNs depending on its location to be placed. Two or three methods can be clubbed with respect to the available expenses to form a hybrid system to meet the energy requirements. The harvested energy can be utilized directly for sensor units and transmission units. Sometimes the harvested energy will be utilized through a battery unit for avoiding supply fluctuations. Most of the time the hybrid systems are added the battery unit for energy regulation purpose to cover up the need of supply to the WSNs.

The energy utilization of the WSNs was controlled by designing a self-healing unstable topology structure [2] for maintain the mobility sensor systems. Here the sensor nodes performances are analyzed by making it as delay torrent networks. The designed structure is helpful in identifying the work continuity of the networks connected between the sensor nodes. A time division multiple access [3] manner is used for WSNs fixed inside a city limit to harvest energy form radio frequencies in an efficient manner. This manner transmit the signals received by the sensor nodes after getting the required energy from the sink nodes. Based on machine learning algorithm a routing technique [4] was proposed to find out the shortest path for information sharing from a source station to the destination part. It saves the energy requirement of for the sensor nodes along with the transmission time. Due to working time minimization of the sensor nodes, the proposed system requires only less power for network operations. 
Journal of Electrical Engineering and Automation (EEA) (2020)

Vol.02/ No. 01

Pages: $13-24$

https://www.irojournals.com/iroeea

DOI: https://doi.org/10.36548/jeea.2020.1.002

A modified dynamic routing source in combination of adaptive routing protocol [5] was introduced to minimize the energy consumption by avoiding the misroute paths of the sensor nodes in WSNs. The system also regulates the drop rates of the pockets transmitted from the source point to destination unit. A fuzzy rule system with concatenation of clustering and neural [6] to determine a shortest path for transmitting the sensor node signals to the IoT unit. The shortest path estimation saves the energy requirement for transmitting the sensor signals. By sending the signals through a shorter way will be helpful in extending the life of installed WSN systems. A WSN node is made with an inbuilt energy harvesting system [7] as an additional unit for providing power supply continuously. The added unit is suggested for sensor networks which are placed very far and unreachable area. The unit minimizes the cost for battery replacement process and delay occurrence due to drain battery. The unit can be fixed with or without battery depending upon the number of sensor nodes connected to the system.

A software defined network system [8] made a multiple path to the destination system to transmit the data securely and effectually. The software defined system reduces the dropping rate by making a multiple path and that multiple paths are always in a dead mode and it will be active when the information is ready to send. Hence the energy requirement for the node transmission is managed by the inbuilt software. A reverse tracking algorithm for smart sensors [9] was framed to minimize the energy consumption of the sensor nodes by rearranging the non-working head nodes in the WSNs. The work also implemented with a scheduling system for utilizing the WSN in an efficient manner to save drop rate and energy. To overcome the same drawbacks in personal communication systems, a cluster backbone approach [10] was developed. Here the virtual cluster structure makes a localized computation for detecting the connectivity issues and shortest path between the source and destination nodes.

An energy balancing system based on pseudo random algorithm [11] is combined with ant architecture to make an efficient route for sensor nodes to transmit the signals. The developed algorithm also accommodates the heuristic algorithm especially for energy management scheme. The pseudo random algorithm works to balance the data to be transmitted on a defined route in WSNs. An optimized route detection technique [12] with respect to soft computing algorithm is structured to overcome the improper routing strategies in WSNs. A fuzzy petri net model is added here to compare and analysis the existing evolutionary algorithms to identify a best route for transmission. This improves the life span of the WSNs along with managing the energy consumption of the sensor nodes. A clustering approach [13] was implemented to improve the energy efficiency in WSNs. In clustering nodes a group of nodes will be added to a common node which acts a head to communicate the data signals to the base stations. A systemized clustering structure makes an order for transmitting the sensed data as one by one to the destination center to save the energy consumption.

The block chain management algorithm [14] makes a trustable network for transmitting the sensor node signal with the help of nearby network device. To secure the transmitting information the block chain 
Journal of Electrical Engineering and Automation (EEA) (2020)

Vol.02/ No. 01

Pages: $13-24$

https://www.irojournals.com/iroeea

DOI: https://doi.org/10.36548/jeea.2020.1.002

network prevents the hacking mechanism involved in the nearby network device and it makes the nearby device as a fly wire network. The block chain network also confirms the packet delivery information to the source unit for saving the energy requirement for transmission. A piezo electric sensor was deployed to make an energy harvesting WSNs [15] to make it as an independent system in source station. With the help of piezo sensor the proposed system was able to produce $3.2 \mathrm{~mW}$ power with reduced sleep time current of $0.95 \mu \mathrm{A}$ to the source circuit and so that the energy harvesting WSNs makes the system in active mode for $1.5 \mathrm{~s}$ longer than its usual time. So that the system was able to send 388bytes a quite large data to the destination unit. An improved flow schedule management practice [16] was employed to manage the IoT networks on its data transmission process when it is employed very near to the other IoT networks. The proposed management system schedules the network data transmission time in an order to avoid data loss and extra energy consumption.

A piezo electric nanogenerator based energy harvesting method [17] was proposed to meet the power supply requirement of the sensor employed in the WSNs. The system achieves a better success rate when it is implemented with an efficient routing protocol. A hybrid genetic algorithm [18] was directed to manage the routing path of the wireless adhoc network by finding a shortest path for signal transmission. The genetic algorithm is combined with a fuzzy logic algorithm for analyzing the packet delivery status of the sensor nodes connected in the network. A solar energy harvesting WSNs [19] with clustering routing methods are implemented to make an efficient and independent network for signal movement. A centralized multi hop routing method was added to this approach for saving the energy required for transmission purpose. A self -power WSNs [20] based on thermal energy harvesting method was introduced to compensate the energy requirement of the sensor nodes fixed in an industrial applications. Here the measured temperature values are transmitted with a unique boost circuit to meet the power requirement of the commercial WSNs. The figure 2 implicates the requirement of an efficient WSNs in terms of energy design and algorithm design. . 
Journal of Electrical Engineering and Automation (EEA) (2020)

Vol.02/ No. 01

Pages: $13-24$

https://www.irojournals.com/iroeea

DOI: https://doi.org/10.36548/jeea.2020.1.002

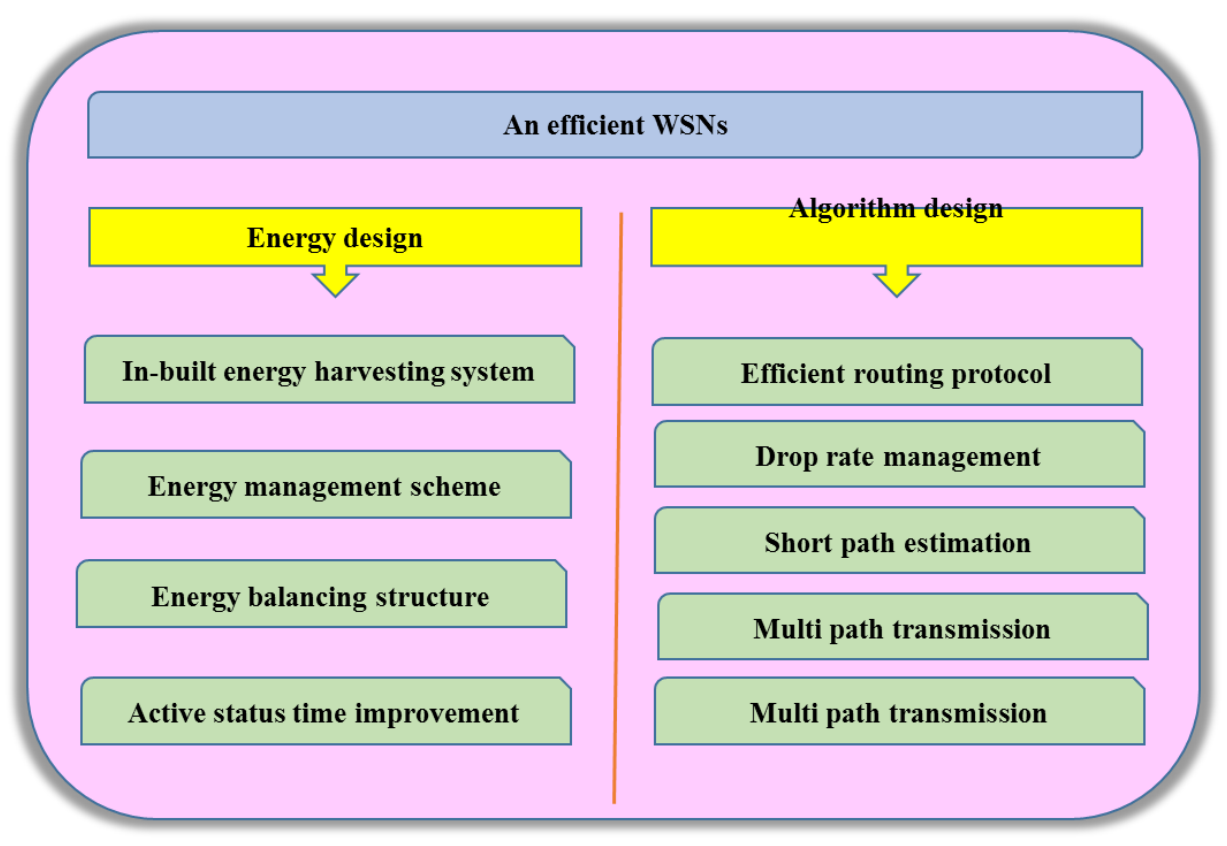

Figure 2. Efficient WSNs requirements

\section{Proposed method}

An efficient WSNs must contains a good energy design for meeting the energy requirement and a good algorithm design to save the energy consumption. The proposed hybrid energy management system concentrates only on the energy design portion of the WSNs to provide a better energy balancing structure for the WSNs fixed in remote areas. Mostly the agriculture lands are be there in remote areas without energy supply. When there is no energy supply, sensor nodes fixed to monitor the agriculture field has to be fixed with an independent energy harvesting system. The general energy harvesting methods for WSNs consists of one or more harvesting techniques to meet the energy requirement based on the availability of sources. The proposed system comprised with natural source and artificial source for making a hybrid system, it consists of Photovoltaic Cell (PC) to pick up energy from the sunlight on the field and Piezo Sensor (PS) for collecting the energy source from the vibration generated on the agriculture field during the water flow. Along with that a Thermal Sensor (TS) is added to it to gain energy from the heated surface on the agriculture field and a simple flow system (FS) is employed to accumulate energy from water and wind source available in the agriculture field. 
Journal of Electrical Engineering and Automation (EEA) (2020)

Vol.02/ No. 01

Pages: $13-24$

https://www.irojournals.com/iroeea

DOI: https://doi.org/10.36548/jeea.2020.1.002

The system is connected with a Soil Moisture Sensor (SMS), Soil Temperature Sensor (STS) for equating the status of the field to the base station and Air Temperature Sensor (ATS) and air humidity sensor for associating the atmosphere changes to the irrigation system placed in the field. The proposed system motive is to meet the energy requirement of all these sensors and the source station as given in the pictorial representation in the figure 3.

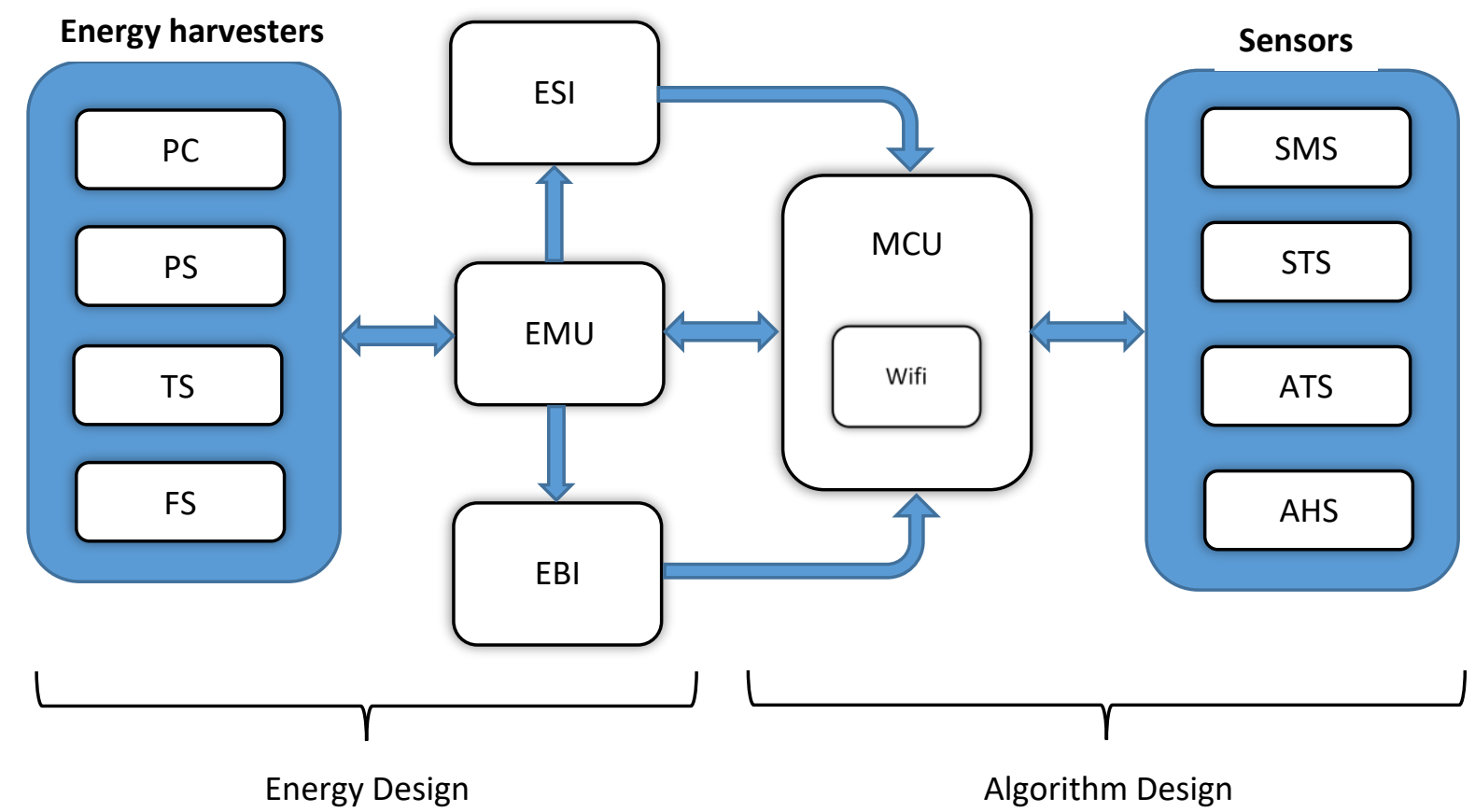

Figure 3. Block diagram of the proposed hybrid energy management system

The proposed energy management system consists of energy harvesters to meet the energy requirement and sensor columns are placed to sense and send the measured values to the Micro-Controller Unit (MCU) with an inbuilt Wifi module for wireless transmission of data to the destination protocol. The system has an arduino nano as an Energy Management Unit (EMU) for transmitting the harvested energy to the Energy Storage Interface (ESI) which can be a battery column and Energy Balancing Interface (EBI) for sharing the load requirement. The EBI are will be a group of capacitors which can be drained during sudden surge requirement of the load system. An arduino microcontroller unit is fixed between the sensor modules and energy management system to maintain the whole process. The power requirement for the microcontroller is 5 Volts with $400 \mathrm{~mA}$ current to perform the required task. So the ESI unit is made to give supply of $6 \mathrm{~V}$ as an output to the microcontroller board and the board regulates the supply from $6 \mathrm{~V}$ 
Journal of Electrical Engineering and Automation (EEA) (2020)

Vol.02/ No. 01

Pages: $13-24$

https://www.irojournals.com/iroeea

DOI: https://doi.org/10.36548/jeea.2020.1.002

to $5 \mathrm{~V}$ by an internal circuit in it. The EBI is an additional ESI unit acts only when there is a complete drain in ESI unit.

Along with sending the sensor signals to the destination station, the system also sends the status of the ESI when it is completely drained. After that the EBI will take of the power supply part to maintain the system operation. The ESI is placed with replacement approach to maintain the continuous operation when there is no power on both EBI and the present ESI unit. The system goes to sleep mode when the action is going EBI power source for extending the standby time of the WSNs. Instead of monitoring the surrounding in a continuous manner the system will monitor in a time space for avoiding the dead operation of the WSNs. This is achieved by adding a timed wakeup protocol to the microcontroller unit. The figure 4 exposes the operation process of the proposed energy management system.

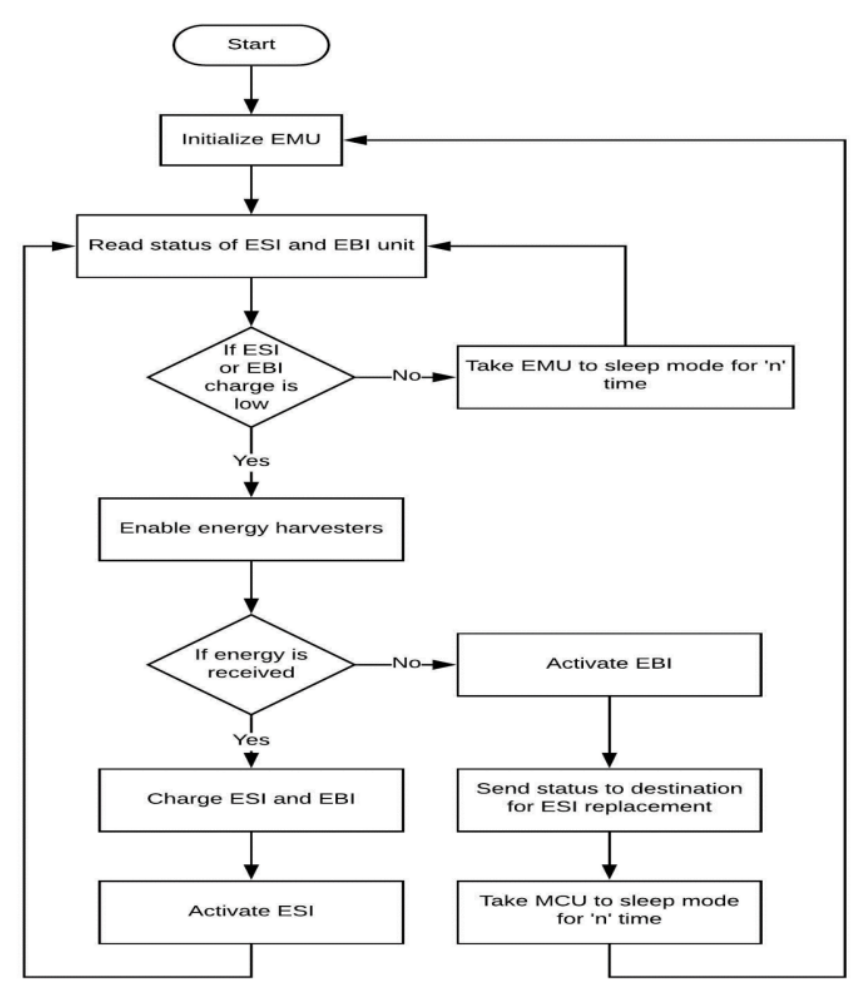

Figure 3. Flow diagram of the proposed energy management system 
Journal of Electrical Engineering and Automation (EEA) (2020)

Vol.02/ No. 01

Pages: $13-24$

https://www.irojournals.com/iroeea

DOI: https://doi.org/10.36548/jeea.2020.1.002

\section{Results and Discussion}

The proposed energy management system is designed to meet the energy requirement of WSNs as well as the requirement of charging the ESI and EBI. As the microcontroller is the brain and head of the whole WSN and the EMU, the proposed technique is designed to meet the energy needed for microcontroller to its maximum current $\left(\mathrm{I}_{\max }\right)$. But the current for driving the network sensors are given in the table 1 .

$$
\mathrm{I}_{\max }=400 \mathrm{~mA}
$$

Table 1. Current rating of the connected system

\begin{tabular}{|c|c|c|}
\hline S.No & Sensor & Rated current $\left(\mathrm{I}_{\mathrm{opr}}\right)$ \\
\hline 1 & SMS (I Isms) & $35 \mathrm{~mA}$ \\
\hline 2 & STS (I $\left.\mathrm{I}_{\mathrm{sts}}\right)$ & $100 \mu \mathrm{A}$ \\
\hline 3 & $\operatorname{ATS}\left(\mathrm{I}_{\mathrm{ats}}\right)$ & $100 \mu \mathrm{A}$ \\
\hline 4 & AHS (Iahs) & $300 \mu \mathrm{A}$ \\
\hline 5 & Wifi shield $\left(\mathrm{I}_{\mathrm{ws}}\right)$ & $183 \mathrm{~mA}$ \\
\hline
\end{tabular}

Therefore the current required for regular operation of the WSN circuit $=\sum I_{o p r}=218.5 \mathrm{~mA}$;

The energy difference between the maximum current and operational current is,

$$
\mathrm{I}_{\mathrm{dif}}=\mathrm{I}_{\max }-\sum I_{\text {opr }}=181.5 \mathrm{~mA} ;
$$

From the $\mathrm{I}_{\max }$ and $\sum I_{\text {opr }}$ the working time $\left(\mathrm{T}_{\mathrm{opr}}\right)$ of the ESI can be calculated. $\mathrm{T}_{\mathrm{opr}}$ is expressed as total capacity of the ESI ( $\left.\mathrm{ESI}_{\text {cap }}\right)$ to the average current consumption by WSN $\left(\mathrm{I}_{\text {avg }}\right)$ in to external factors which is a constant $(\alpha)$.

$$
\mathrm{T}_{\mathrm{opr}}=\frac{E S I_{\text {cap }}}{I_{\text {avg }}} * \alpha
$$

Eq. 1 
Journal of Electrical Engineering and Automation (EEA) (2020)

Vol.02/ No. 01

Pages: $13-24$

https://www.irojournals.com/iroeea

DOI: https://doi.org/10.36548/jeea.2020.1.002

Similarly, the operation of the proposed system can be calculated for its maximum energy consumption $\mathrm{I}_{\max }$ as expressed in equation 2 .

$\mathrm{T}_{\mathrm{opr}}=\frac{E S I_{\text {cap }}}{I_{\max }} * \alpha$

Eq. 2

Where, $\alpha=0.7$

The connected ESI and EBI unit consist nickel metallic hydride battery of 6V power package with $1300 \mathrm{mAh}$ battery source for the proposed energy management operation. The ESI and EBI are charged to $1 / 10$ of its total capacity current and it needs 16 hours of time to charge fully which is calculated as,

ESI \& EBI charging $\left(\mathrm{I}_{\text {chg }}\right)=\frac{\text { total battery pack rated current }}{10}=\frac{1300+1300}{10}=260 \mathrm{mAh}$;

Therefore the total current ( $\left.\mathrm{I}_{\mathrm{tot}}\right)$ requirement from the energy harvesters is the summation of operation current to the charging current needed for the ESI and EBI and it is expressed in the equation 3.

$I_{\text {tot }}=\sum I_{o p r}+I_{c h g}$

Eq.3

So,

$$
I_{\text {tot }}=218.5+260=478.5 \sim 500 \mathrm{~mA}
$$

$I_{\text {tot }}$ indicates the required current needed from the energy harvester column and the voltage rating of the power bank is $6 \mathrm{~V}$ so that the voltage needed for charging is 1.5 time of the power bank voltage and it will be $9 \mathrm{~V}$. Hence, the energy to be generated by the energy harvester is $6 \mathrm{~V}, 500 \mathrm{~mA}$. So a solar panel, piezo sensor column, thermal sensor and flow sensors of the same voltage and current is fixed in the energy management system. The solar panel can able to generate power efficiently for four hours a day. Similarly the piezo sensors fixed to pick up generation from water flow which are able to generate power maximum 2 hours a day. Thermal system can generate supply for 6 hours a day and the flow system can generate 2 hours from water flow and 3 hours from air flow in day and night time. As the energy harvesters are connected in series to each other to gain current in generation for faster charging. 
Journal of Electrical Engineering and Automation (EEA) (2020)

Vol.02/ No. 01

Pages: $13-24$

https://www.irojournals.com/iroeea

DOI: https://doi.org/10.36548/jeea.2020.1.002

The average operational time current of the proposed energy management system can be calculated from $\sum I_{\text {opr }}$ value to the following equation 4.

$\mathrm{I}_{\mathrm{opr}}=\frac{\left(\text { oN }_{\text {time }} \cdot \sum I_{\text {opr }}+\text { Sleep }_{\text {time }} \cdot \text { Sleep }_{\text {current }}\right)}{\left(\text { ON }_{\text {time }}+\text { Sleep }_{\text {time }}\right)}$

Eq. 4

$O N_{\text {time }}($ WSNs on time $)=250 \mathrm{mSecond}=0.25$ Seconds,

Sleep $_{\text {time }}($ WSNs off time $)=30$ Minutes $=1800$ Seconds,

Sleep $_{\text {current }}=\sim 100 \mu \mathrm{A}$,

Therefore,

$$
\mathrm{I}_{\mathrm{opr}}=0.130 \mathrm{~mA}
$$

$\therefore$ Average power of the system is $\mathrm{P}_{\mathrm{avg}}=\mathrm{V}^{*} \mathrm{I}_{\mathrm{opr}}$

$$
\mathrm{V}=\text { Rated voltage of microcontroller }=5 \text { Volts }
$$

So, $\quad \mathrm{P}_{\mathrm{avg}}=0.65 \mathrm{~mW}$;

The ESI unit capacity $=1300 \mathrm{mAh}$; with voltage of $5 \mathrm{~V}$; and the Power $(\mathrm{P})=6500 \mathrm{mWh}$.

Hence, the life of ESI to operate $=\frac{P}{P_{\text {avg }}}=10000$ hours $\sim 13$ months.

Therefore the proposed system can operate continuously in sensing and data transmission through WSNs for 13 months approximately. The balancing unit EBI is useful in sudden surge requirement of power and in drain condition due to failure of solar cell and any other energy harvester unit. The sleep time of the WSNs can be modified in the algorithm process which can change the life span of the ESI block to reach 
Journal of Electrical Engineering and Automation (EEA) (2020)

Vol.02/ No. 01

Pages: $13-24$

https://www.irojournals.com/iroeea

DOI: https://doi.org/10.36548/jeea.2020.1.002

drain without charging. The energy harvesters makes a way to improve the WSNs with additional sensors for detection.

\section{Conclusion}

The proposed hybrid energy management system for WSNs in remote areas can able to send sensor signals to the destination station continuously for 13 months in the regular interval of 30minutes once for every 24 hours. The system is employed with an additional EBI unit for energy balancing purpose and it also helpful when the ESI unit is drained due to environmental changes. The system is designed for $500 \mathrm{~mA}$ load circuit for supplying the charging unit as well as the WSNs blocl. The proposed system can be fixed anywhere when there is no power supply available for the WSNs. A combination of good algorithm design with the proposed system will make the whole WSN as an effective one.

\section{Reference}

[1] Koresh, H. James Deva, and J. Vijitha Ananthi. "Simulation of sensor device using battery replacement approach." In 2017 International Conference on Inventive Computing and Informatics (ICICI), pp. 758-761. IEEE, 2017.

[2] Smys, S., and Jennifer S. Raj. "A self-organized structure for mobility management in wireless networks." Computers \& Electrical Engineering 48 (2015): 153-163.

[3] Liu, Jingxian, Ke Xiong, Pingyi Fan, and Zhangdui Zhong. "RF energy harvesting wireless powered sensor networks for smart cities." IEEE Access 5 (2017): 9348-9358.

[4] Duraipandian, M. "Performance evaluation of routing algorithm for Manet based on the machine learning techniques." Journal of trends in Computer Science and Smart technology (TCSST) 1, no. 01 (2019): 25-38.

[5] Kavitha, M., and B. G. Geetha. "An efficient city energy management system with secure routing communication using WSN." Cluster Computing 22, no. 6 (2019): 13131-13142.

[6] Raj, Jennifer S. "QoS optimization of energy efficient routing in IoT wireless sensor networks." Journal of ISMAC 1, no. 01 (2019): 12-23.

[7] Gupta, Sakar, and Sunita Gupta. "Analysis and Comparison of Sensor Node Scheduling Heuristic for WSN and Energy Harvesting WSN." In Smart Systems and IoT: Innovations in Computing, pp. 131-139. Springer, Singapore, 2020.

[8] Anand, J. V. "Design and development of secure and sustainable software defined networks." Journal of Ubiquitous Computing and Communication Technologies (UCCT) 1, no. 02 (2019): 110-120. 
Journal of Electrical Engineering and Automation (EEA) (2020)

Vol.02/ No. 01

Pages: $13-24$

https://www.irojournals.com/iroeea

DOI: https://doi.org/10.36548/jeea.2020.1.002

[9] Hemalatha, P., J. Vijitha Ananthi, and R. Kalaivani. "Analysis of reverse tracing algorithm for the detection of DOS attacks in MANET." International Journal of Autonomic Computing 2, no. 4 (2017): 311-322.

[10] Smys, S., and G. Josemin Bala. "Efficient self-organized backbone formation in mobile ad hoc networks (MANETs)." Computers \& Electrical Engineering 38, no. 3 (2012): 522-532.

[11] Li, Xinlu, Brian Keegan, Fredrick Mtenzi, Thomas Weise, and Ming Tan. "Energy-Efficient Load Balancing Ant Based Routing Algorithm for Wireless Sensor Networks." IEEE Access 7 (2019): 113182-113196.

[12] Haoxiang, Wang, and S. Smys. "Soft Computing Strategies for Optimized Route Selection in Wireless Sensor Network." Journal of Soft Computing Paradigm (JSCP) 2, no. 01 (2020): 112.

[13] Cacciagrano, Diletta, Rosario Culmone, Matteo Micheletti, and Leonardo Mostarda. "Energyefficient clustering for wireless sensor devices in internet of things." In Performability in Internet of Things, pp. 59-80. Springer, Cham, 2019.

[14] Bhalaji, N. "QOS and defense enhancement using block chain for fly wireless networks." Journal of trends in Computer Science and Smart technology (TCSST) 1, no. 01 (2019): 1-13.

[15] Ruan, Tingwen, Zheng Jun Chew, and Meiling Zhu. "Energy-aware approaches for energy harvesting powered wireless sensor nodes." IEEE Sensors Journal 17, no. 7 (2017): 21652173.

[16] Pandian, M. Durai. "Enhanced network performance and mobility management of IOT multi networks." Journal of trends in Computer Science and Smart technology (TCSST) 1, no. 02 (2019): 95-105.

[17] Anisi, Mohammad Hossein, Gaddafi Abdul-Salaam, Mohd Yamani Idna Idris, Ainuddin Wahid Abdul Wahab, and Ismail Ahmedy. "Energy harvesting and battery power based routing in wireless sensor networks." Wireless Networks 23, no. 1 (2017): 249-266.

[18] Rahimunnisa, K. "Hybridized genetic-simulated annealing algorithm for performance optimization in wireless adhoc network." Journal of Soft Computing Paradigm (JSCP) 1, no. 01 (2019): 1-13.

[19] Bozorgi, Seyed Mostafa, Ali Shokouhi Rostami, Ali Asghar Rahmani Hosseinabadi, and Valentina Emilia Balas. "A new clustering protocol for energy harvesting-wireless sensor networks." Computers \& Electrical Engineering 64 (2017): 233-247.

[20] Hou, Liqun, Shudong Tan, Zhijuan Zhang, and Neil W. Bergmann. "Thermal energy harvesting WSNs node for temperature monitoring in IIoT." IEEE Access 6 (2018): 35243-35249. 